
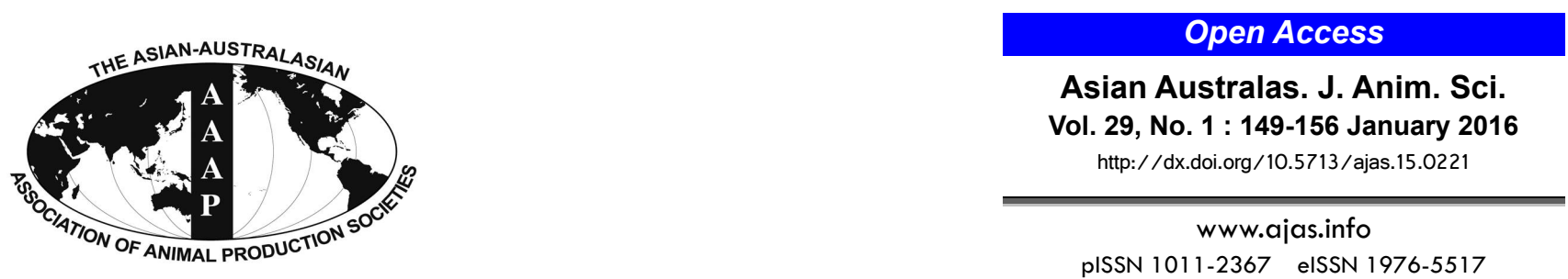

\title{
Monitoring Activity for Recognition of Illness in Experimentally Infected Weaned Piglets Using Received Signal Strength Indication ZigBee-based Wireless Acceleration Sensor
}

\author{
Sonia Tabasum Ahmed, Hong-Seok Mun, Md. Manirul Islam, Hyun Yoe ${ }^{1}$, and Chul-Ju Yang* \\ Department of Animal Science and Technology, Sunchon National University, Suncheon 540-950, Korea
}

\begin{abstract}
In this experiment, we proposed and implemented a disease forecasting system using a received signal strength indication ZigBee-based wireless network with a 3-axis acceleration sensor to detect illness at an early stage by monitoring movement of experimentally infected weaned piglets. Twenty seven piglets were divided into control, Salmonella enteritidis (SE) infection, and Escherichia coli (EC) infection group, and their movements were monitored for five days using wireless sensor nodes on their backs. Data generated showed the 3-axis movement of piglets (X-axis: left and right direction, Y-axis: anteroposterior direction, and Z-axis: up and down direction) at five different time periods. Piglets in both infected groups had lower weight gain and feed intake, as well as higher feed conversion ratios than the control group $(\mathrm{p}<0.05)$. Infection with $\mathrm{SE}$ and EC resulted in reduced body temperature of the piglets at day 2,4 , and $5(\mathrm{p}<0.05)$. The early morning $\mathrm{X}$-axis movement did not differ between groups; however, the $\mathrm{Y}$-axis movement was higher in the EC group (day 1 and 2), and the Z-axis movement was higher in the EC (day 1) and SE group (day 4) during different experimental periods $(\mathrm{p}<0.05)$. The morning $\mathrm{X}$ and $\mathrm{Y}$-axis movement did not differ between treatment groups. However, the Z-axis movement was higher in both infected groups at day 1 and lower at day 4 compared to the control $(\mathrm{p}<0.05)$. The midday $\mathrm{X}$-axis movement was significantly lower in both infected groups (day 4 and 5) compared to the control $(\mathrm{p}<0.05)$, whereas the Y-axis movement did not differ. The Z-axis movement was highest in the SE group at day 1 and 2 and lower at day 4 and $5(p<0.05$ ). Evening $\mathrm{X}$-axis movement was highest in the control group throughout the experimental period. During day 1 and 2 , the Z-axis movement was higher in both of the infected groups; whereas it was lower in the SE group during day 3 and $4(p<0.05)$. During day 1 and 2 , the night $\mathrm{X}$-axis movement was lower and the $\mathrm{Z}$-axis movement was higher in the infected piglets $(\mathrm{p}<0.05)$. Overall, the movement of infected piglets was altered, and the acceleration sensor could be successfully employed for monitoring pig activity. (Key Words: Acceleration Sensor, Pig Activity, Bacterial Infection, Wireless Sensor Network)
\end{abstract}

\section{INTRODUCTION}

Infection with enteric pathogens such as Escherichia coli (E. coli) or Salmonella is common in intensive pig farming and associated with poor performance and animal welfare (Pijpers et al., 1991; Greiner et al., 2000), as well as expensive veterinarian intervention costs. The cost and effectiveness of eradication or control of a disease outbreak is normally directly related to delays in detection. Moreover,

\footnotetext{
* Corresponding Author: Chul-Ju Yang. Tel: +82-61-750-3235, Fax:+82-61-750-3239, E-mail: yangcj@scnu.kr

${ }^{1}$ Department of Information and Communication Engineering, Sunchon National University, Suncheon 540-950, Korea.

Submitted Mar. 17, 2015; Revised May 8, 2015; Accepted Jun. 3, 2015
}

it is known that detecting animal illness and providing both individual care and group-by-group mass therapy is not very effective, despite its high costs. Since infection is primarily spread via horizontal transmission from carrier animals to the others in close contact (Schwartz, 1991), the best way to handle an illness is early detection of diseases and treatment of the animal before the disease spreads.

Over the past few decades, the relationship between behavior and animal health has gained attention (Weary et al., 2009). Several studies have demonstrated that the behavior and physiological responses of farmed animals provide reliable information about animal health status and welfare (Mepham, 2000; Nardone et al., 2004). For example, increased or decreased overall movement within a group 
may imply an emerging disease. Surveillance of animal disease can serve as sentinels for bioterrorist or natural infectious disease epidemics. Pigs exposed to pathogenic bacteria, such as enteric E. coli or Salmonella strains often exhibit altered behaviors in terms of activity (Ivoš et al., 1981). Behavioral monitoring in terms of activity should enable development of methodologies for detection of individuals with potential health problems, which could have a significant impact on practical farming. Traditionally, such evaluations have been based on observation of activity with the naked eye. However, in large groups of pigs, it is very difficult to visually measure behavior and monitor the health of individual pigs, let alone their welfare and movement. The development of systems for automatic monitoring of animal behavior has become possible owing to the evolution of new sensor technologies. Indeed, the invention of wireless sensor networks has led to a new level of possibilities in this area, with the potential for measuring animal behavior in natural and undisturbed environments. ZigBee is a $2.4 \mathrm{GHz}$-based low cost, low power, low data rate wireless technology built on top of the IEEE 802.15.42003 standard. A number of studies have demonstrated the successful use of ZigBee received signal strength indication (RSSI)-based wireless sensor networks to monitor and classify animal behavior (Nadimi et al., 2012).

Accelerometers are sensors that can be used as motion detectors (DeVaul and Dunn, 2001), as well as for bodyposition and posture sensing (Foerster et al., 1999). Since they respond to both acceleration due to movement and gravitational acceleration, body-mounted accelerometers equipped with wireless interfaces show great promise for use in remote sensing of the behavior of individuals based on their movement. In the present study, we evaluated use of an acceleration sensor livestock disease surveillance system composed of the RSSI ZigBee-based wireless network to register the activity of piglets experimentally infected with Salmonella enteritidis (S. enteritidis) or $E$. coli. The overall objective was to investigate to what extent physical activity/movement changes in response to oral infection with $S$. enteritidis and $E$. coli, and to establish whether or not monitored behavior altered due to infection can be used as an early sign of pending disease induced by inoculated bacteria.

\section{MATERIALS AND METHODS}

\section{System design}

In this study, we evaluated an acceleration sensor livestock disease surveillance system using RSSI ZigBeebased wireless network. The system consists of an active tag acceleration sensor, a universal serial bus (USB) type receiver and a computer (data acquisition system) (Figure 1). The active tag consisting of a low power 3-axis accelerometer (Model NT-24AT-PBG; NETHOM, Gunpo, Korea) operating at a $2.4 \mathrm{GHz}$ ISM (industrial, scientific, and medical) radio frequency band was used to measure acceleration within a range of $\pm 2.5 \mathrm{~g}$ and acted as a signal transmitter with 30 to $100 \mathrm{~m}$ coverage. The acceleration sensor node was run on an AAA Energizer E92 alkaline battery capable of supporting the system for approximately $48 \mathrm{~h}$. The wireless signals were transmitted to a connected computer through a USB type receiver capable of decoding or demodulating the signals. The active tags attached to the piglet could only detect signals when the piglets moved. The activity of the piglets was determined from the data displayed on the computer in table format.

\section{Infection experiment}

This experiment was conducted under the approval of the Sunchon National University Animal Care and Use Committee (SCNU_IACUC_2012-11). A total of 27 weaned piglets (average 4 weeks old with $9.58 \mathrm{~kg}$ average initial weight) were obtained from a high-health status farm (from the same herd) that was reportedly free from all major pathogens of swine, including Salmonella and E. coli. Animals were randomly distributed into three experimental groups composed of three replications with three piglets each in such a way that all groups had a similar mean live weight. The experimental groups were: control, infection with $S$. enteritidis (SE) and infection with $E$. coli (EC). The piglets in each group were reared in an environmentally controlled room in three isolated pens (slatted floor and a

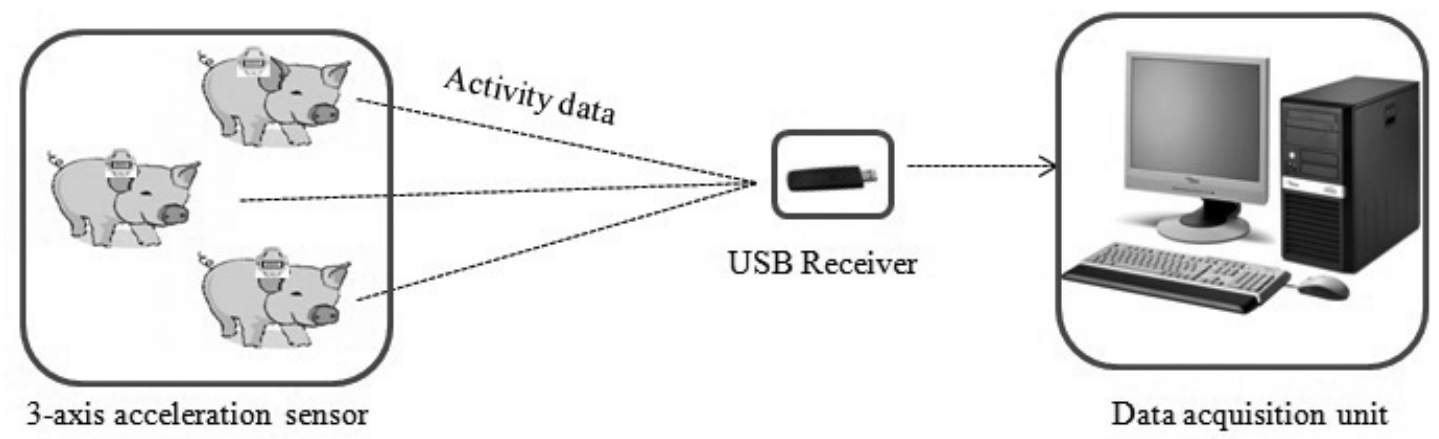

Figure 1. Diagram of the ZigBee-based livestock activity monitoring system. USB, universal serial bus. 
space allowance of $0.75 \mathrm{~m}^{2}$ per piglet) and allowed a 2week adaptation period before commencing the study. Piglets in the SE and EC group were then orally infected with $10 \mathrm{~mL}$ suspensions of either $S$. enteritidis Korean Collection for Type Culture (KCTC) 2021 or E. coli KCTC $2571\left(1 \times 10^{8} \mathrm{cfu} / \mathrm{mL}\right)$. The bacterial strains are part of the KCTC and were purchased from the Korea Research Institute of Bioscience and Biotechnology, Daejeon, Korea. Frozen stock cultures of $S$. enteritidis or E. coli (maintained at $-80^{\circ} \mathrm{C}$ in glycerol) were used to prepare the inocula by growth in nutrient broth (NB) for $24 \mathrm{~h}$ at $37^{\circ} \mathrm{C}$. The piglets in the control group were orally inoculated with $10 \mathrm{~mL}$ of NB. An ambient temperature of $24 \pm 1.5^{\circ} \mathrm{C}$ was maintained in the pig houses via overhanging electric heaters. During the experimental period, piglets were provided with a commercial diet and water on ad libitum basis. The pens were illuminated by artificial light, and ventilation was provided by ten air changes per hour. There was no medication program to avoid effect of stress on pigs' movements.

\section{Clinical observation}

To monitor post-infection performance, body weight gain and feed intake of pigs were recorded on the day of inoculation (day 0) and at the end of the experiment (day 5). Individual piglet's ear temperatures were recorded daily using a Braun ThermoScan ear thermometer (Braun GmbH, Kronberg, Germany). The occurrence and severity of diarrhea were monitored and assessed daily on a pen basis by using a fecal consistency scoring system $(0=$ normal; 1 $=$ soft feces; $2=$ mild diarrhea; $3=$ severe diarrhea) (Marquardt et al., 1999).

\section{Activity observation}

To monitor the movements, all piglets were equipped with wireless acceleration sensor nodes on their back (Figure 2). The weight of the wireless sensor node was about $130 \mathrm{~g}$, including the battery, which was easy for the piglets to carry and was securely tied to pig so that bitten or playing with other pigs impose little effect on the movement reading. We did not find any severe curiosity of piglets with the sensor node. Once activated, the active tag continuously sampled acceleration data in three axes (Xaxis: left and right direction, Y-axis: anteroposterior direction, and Z-axis: up and down direction) and sent data to a nearby $(<30 \mathrm{~m})$ PC computer for analysis and storage using a $2.4 \mathrm{GHz}$ wireless USB type receiver. Analyses were conducted offline after the acceleration data has been collected and transmitted to excel files for further analysis.

\section{Statistical analyses}

All data for each variable were confirmed to be normally distributed. Data for movements were first transformed to absolute values using the ABS option of Microsoft excel to calculate the sum of each axis two hour activity. The sum values were then used to conduct the statistical analysis. Pen of three piglets was considered as the experimental unit. Statistical analyses were conducted using the SAS system ver. 9.1 (SAS Institute Inc., Cary, NC, USA) based on the statistical model:

$$
Y_{i j}=\mu+\alpha_{i}+e_{i j}
$$

Where, $Y_{i j}=$ the response variable, $\mu=$ the general mean, $\alpha_{i}=$ the effect of treatments and $e_{i j}=$ the random error. Duncan's multiple range tests were used to identify significant differences between treatments. All p-values $<0.05$ were considered significant, while values $0.05<\mathrm{p}<0.10$ were considered tendencies.

\section{RESULTS}

The growth performance of weaned piglets in response to infection with SE or EC is shown in Table 1. Five days

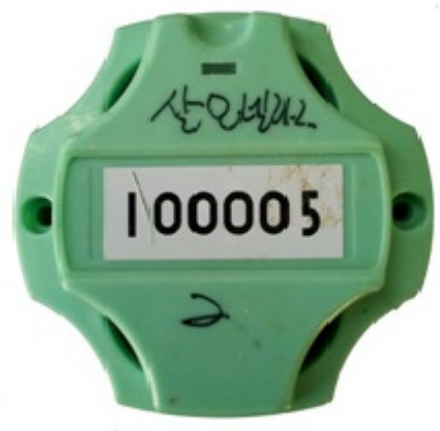

(A)

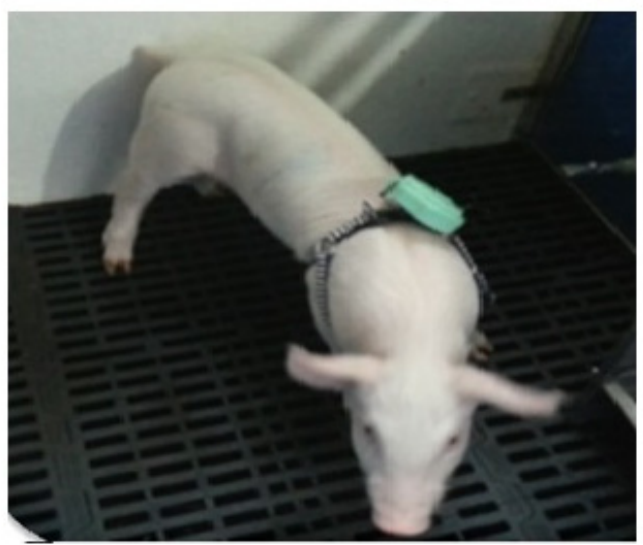

(B)

Figure 2. Acceleration sensor and pig with sensor in their back. (A) Active tag. (B) Piglets with active tag. 
Table 1. Effects of Salmonella enteritidis (SE) and Escherichia coli (EC) infection on the growth performance of weaned piglets ${ }^{1}$

\begin{tabular}{lccccc}
\hline Items & Control & SE & EC & SEM & p-value \\
\hline Day 1 body weight $(\mathrm{kg})$ & 16.50 & 16.38 & 16.24 & 0.22 & 0.7319 \\
Day 5 body weight $(\mathrm{kg})$ & $22.22^{\mathrm{a}}$ & $20.79^{\mathrm{b}}$ & $19.87^{\mathrm{b}}$ & 0.29 & 0.0041 \\
Weight gain (kg) & $5.72^{\mathrm{a}}$ & $4.40^{\mathrm{b}}$ & $3.62^{\mathrm{c}}$ & 0.13 & 0.0002 \\
Feed intake (kg) & $6.90^{\mathrm{a}}$ & $6.13^{\mathrm{b}}$ & $5.65^{\mathrm{b}}$ & 0.21 & 0.0168 \\
FCR (feed/gain) & $1.21^{\mathrm{b}}$ & $1.40^{\mathrm{a}}$ & $1.56^{\mathrm{a}}$ & 0.05 & 0.0069 \\
\hline
\end{tabular}

SEM, standard error of the mean; FCR, feed conversion ratio.

${ }^{1}$ Each value represents the mean of 9 piglets.

${ }^{a, b}$ Means with different superscripts differ significantly at $\mathrm{p}<0.05$.

after infection, the body weight $(\mathrm{p}=0.0041)$ and feed intake $(\mathrm{p}=0.0168)$ of both infected groups were significantly lower than those of the control group. The weight gain was greatly retarded in response to oral infection with $\mathrm{SE}$ or $\mathrm{EC}$, with the lowest gain being observed in the EC infected group ( $p=0.0002)$. The lowest feed conversion ratio was observed in the control group ( $p$ $=0.0069)$. The ear temperature of the piglets in both infected group was lower than control group during day 2, 4, and 5 (Figure 3). Although there was no significant difference, the fecal consistency score of control was measured as normal to soft feces, whereas in infected groups it was normal to mild diarrhea (data not shown).

Figure 4 shows the early morning (4:00 to 6:00 am) activity of weaned piglets up to 5 days post-infection in response to infection with SE or EC. No significant differences were reported for the $\mathrm{X}$-axis movement during the experimental period. The Y-axis movement of the EC infected group was significantly higher than that of the control and SE infected group during day 1 and 2 postinfection $(\mathrm{p}<0.05)$. No differences in the $\mathrm{Y}$ axis movement were recorded for day 3, 4, or 5. The Z-axis movement was also higher in the EC infected group than the control group at day $1(\mathrm{p}=0.0072)$, whereas the highest movement was reported in the SE infected group on day $3(\mathrm{p}=0.0346)$. The morning (8:00 to 10:00 am) $\mathrm{X}$-axis and Y-axis

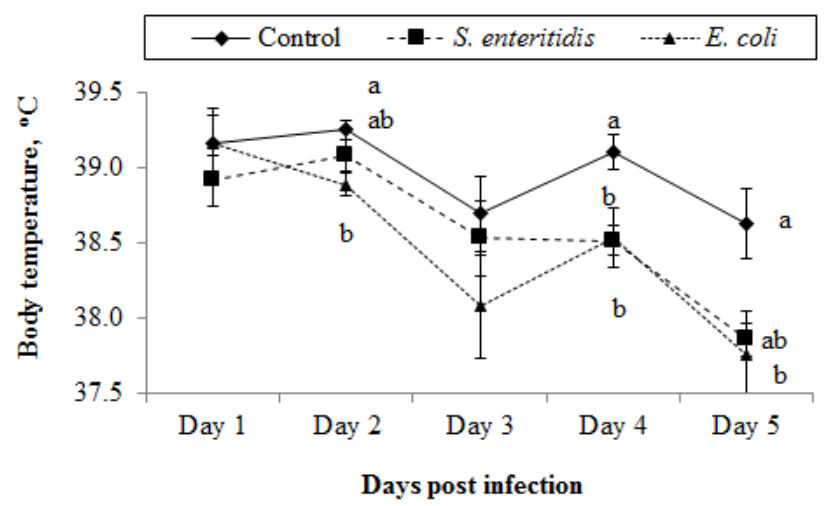

Figure 3. Effects of Salmonella enteritidis and Escherichia coli infection on the ear temperature of weaned piglets. Lines within a time period not sharing a common letter are significantly different $(\mathrm{p}<0.05)$. movement of weaned piglets did not differ between treatment groups during the experimental period (Figure 5). During day 1 , the highest $Z$-axis movement was shown by piglets in both infected groups $(p=0.0486)$. However, at day 4 , the Z-axis movement was highest in the control group $(p=0.0172)$.

The midday (12:00 to $14: 00 \mathrm{pm}) \mathrm{X}$-axis movement of weaned piglets did not differ between treatment groups for

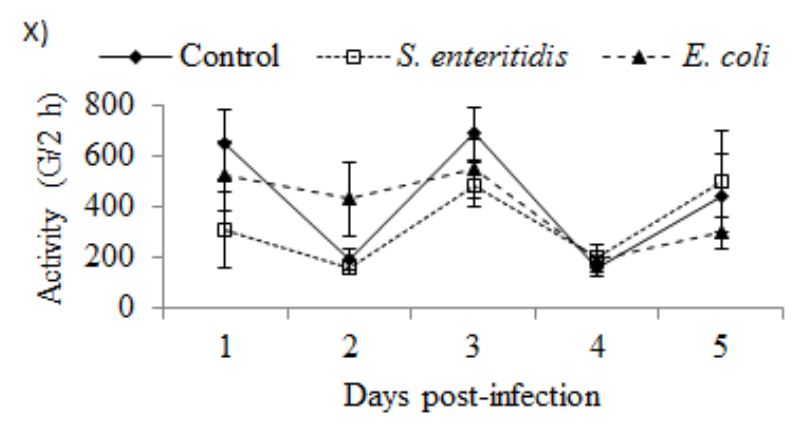

Y) —C Control -.-๒-..S. enteritidis - - - E. coli

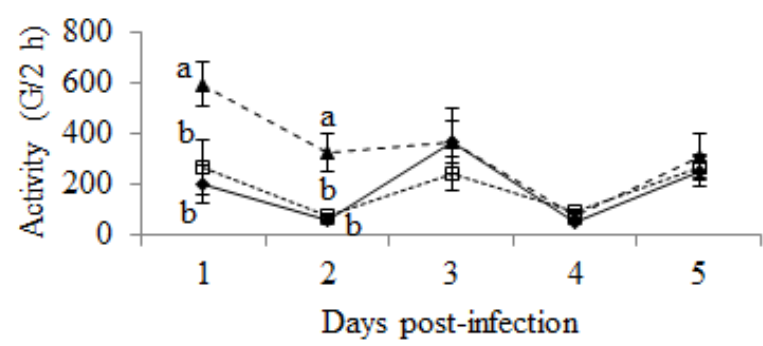

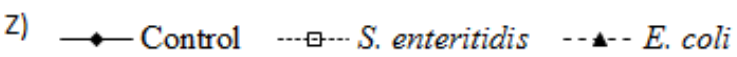

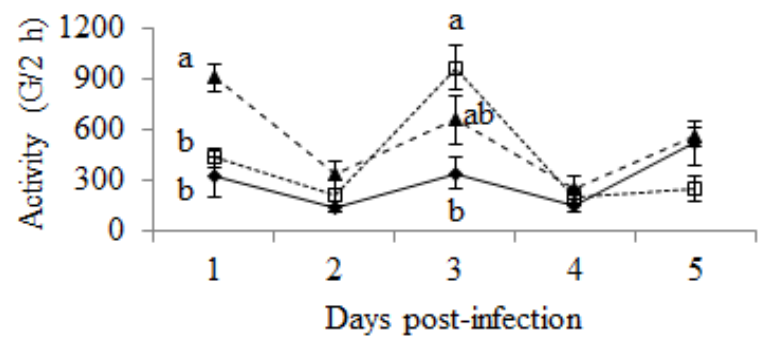

Figure 4. Effects of Salmonella enteritidis and Escherichia coli infection on the early morning (4:00 to 6:00) axial movements (X, $\mathrm{Y}$, and $\mathrm{Z}$ axis) of weaned piglets. Lines within a time period not sharing a common letter are significantly different $(\mathrm{p}<0.05)$. 

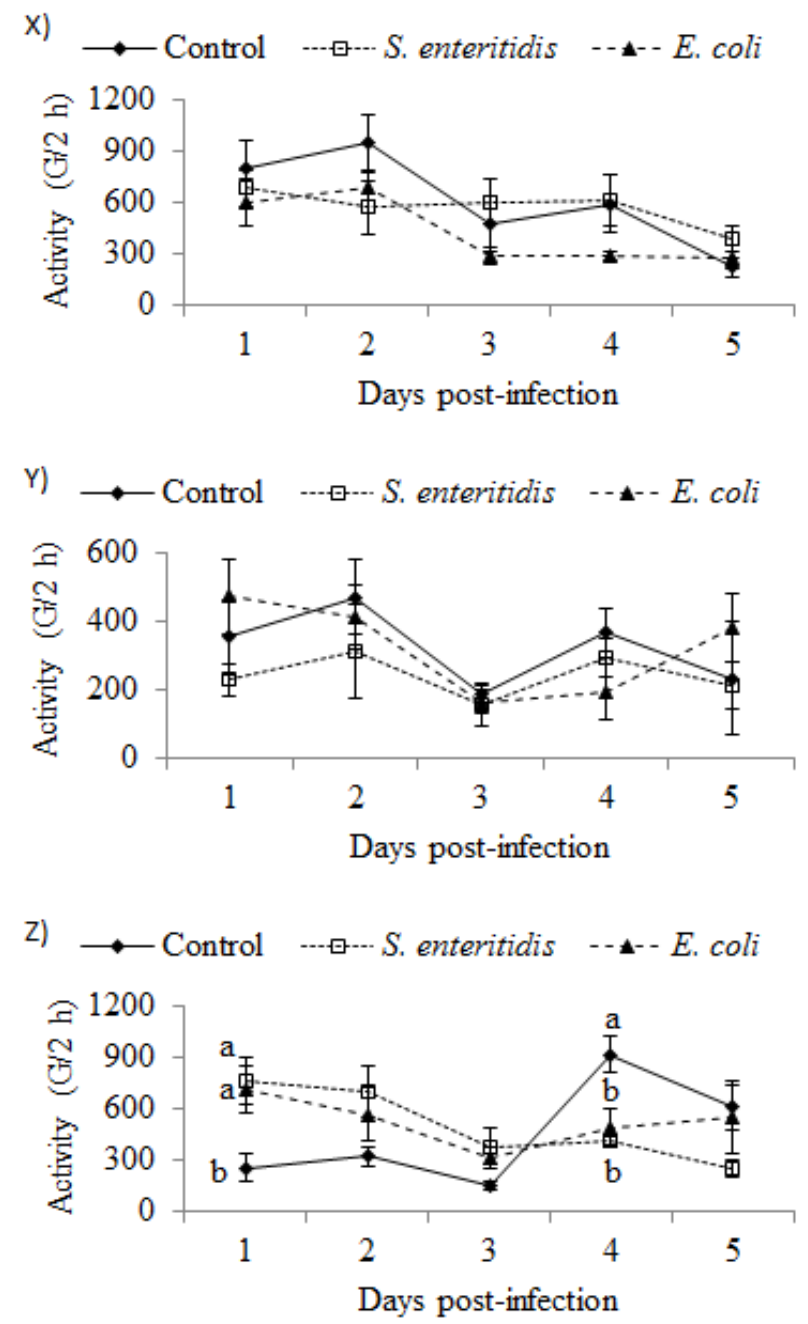

Figure 5. Effects of Salmonella enteritidis and Escherichia coli infection on the morning (8:00 to 10:00) axial movements (X, Y, and $\mathrm{Z}$ axis) of weaned piglets. Lines within a time period not sharing a common letter are significantly different $(\mathrm{p}<0.05)$.

the first 3 days post-infection (Figure 6). During day $4(\mathrm{p}=$ $0.0056)$ and $5(p=0.0731)$, both infected groups showed lower $\mathrm{X}$-axis movement than the control group. No significant difference was recorded for the midday Y-axis movement between treatment groups. During the first two days post-infection, the highest $\mathrm{Z}$-axis movement was recorded in the SE infected group relative to the control $(p<0.05)$, whereas no difference was recorded at day 3 . During day 4 and 5, the SE infected group showed the lowest Z-axis movement compared to the control group $(\mathrm{p}<0.05)$.

During day 1 and 2, the evening (18:00 to 20:00 pm) Xaxis movement of both infected groups was lower $(\mathrm{p}<0.05)$ than that of control group piglets (Figure 7). At day 3, the lowest movement was observed in the EC infected group ( $p$ $=0.0368$ ). On day 4 and 5, both infected groups showed lower $\mathrm{X}$-axis movement than the control group $(\mathrm{p}<0.05)$. There were no significant differences in Y-axis movement
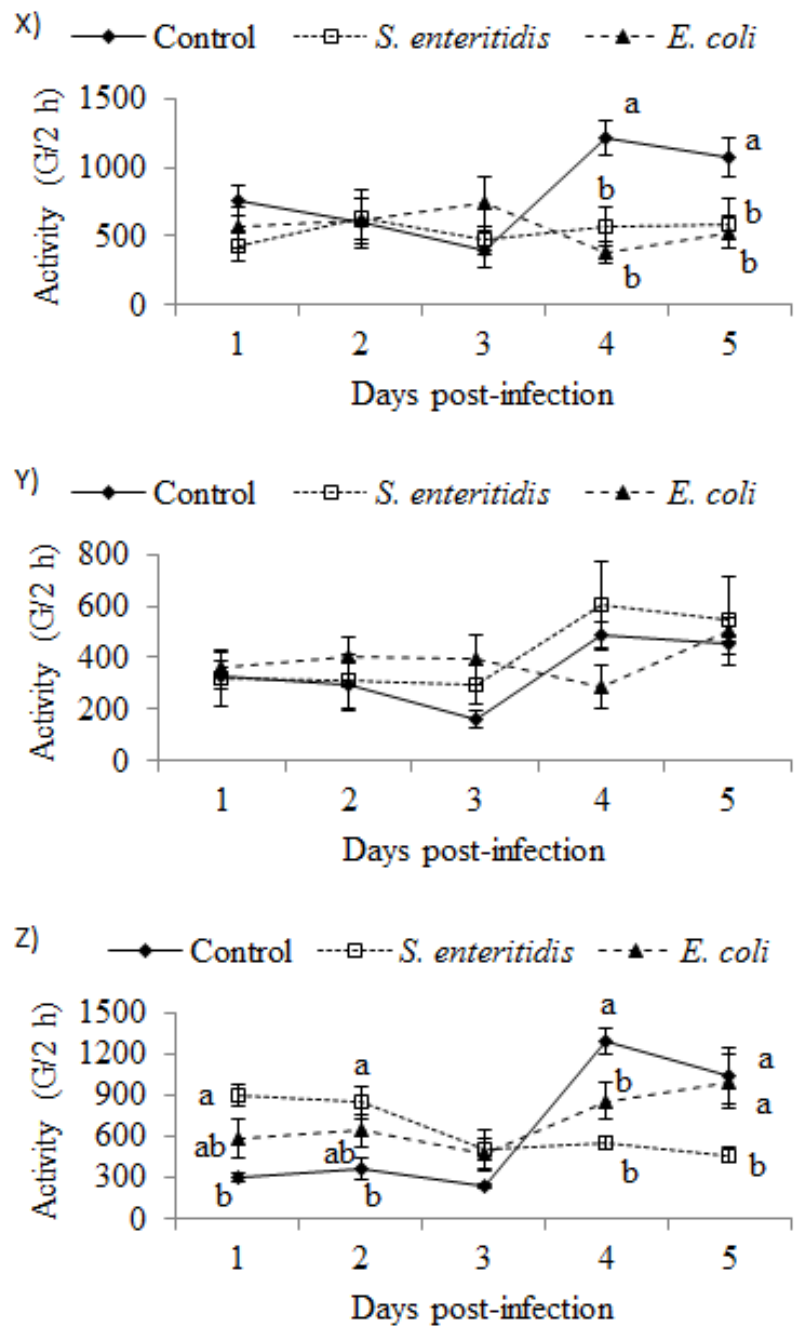

Figure 6. Effects of Salmonella enteritidis and Escherichia coli infection on the midday (12:00 to 14:00) axial movements (X, Y, and $\mathrm{Z}$ axis) of weaned piglets. Lines within a time period not sharing a common letter are significantly different $(\mathrm{p}<0.05)$.

between treatment groups. The evening Z-axis movement was higher in both infected groups of piglets than the control during the first 2 days post-infection $(p<0.07)$, whereas at day 3 and 4, the SE infected group showed lower movement than the other two groups $(p<0.03)$. No significant difference in evening Z-axis movement was observed among groups at day 5 .

At night (22:00 to $24: 00 \mathrm{pm}$ ) on day 1 and 2 , the $\mathrm{X}$-axis movements of both infected groups were lower than those of the control $(p<0.02$, Figure 8$)$. No significant differences among groups were observed on days 3, 4, and 5. Oral infection with SE or EC had no significant effects on Y-axis movement of weaned piglets. During day 1, the highest Zaxis movement was recorded in the EC infected group, whereas both infected groups showed higher movement than the control on day 2. At day 4, the Z-axis movement of EC infected piglets tended to be higher than that of the SE infected group $(p=0.0707)$; however, it did not differ from 

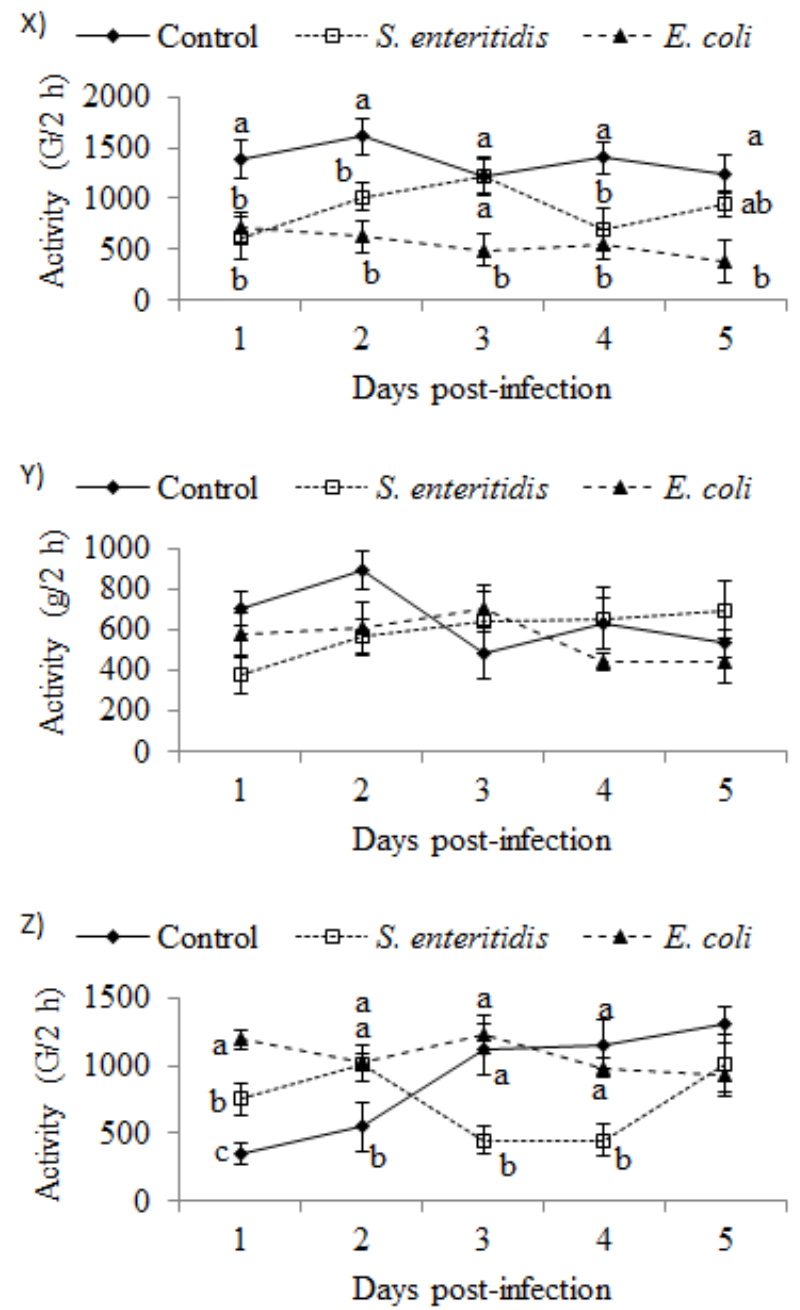

Figure 7. Effects of Salmonella enteritidis and Escherichia coli infection on the evening (18:00 to 20:00) axial movements (X, Y, and $\mathrm{Z}$ axis) of weaned piglets. Lines within a time period not sharing a common letter are significantly different $(p<0.05)$.

the control.

\section{DISCUSSION}

An infection experiment using weaned piglets was conducted to investigate the effects of $S$. enteritidis and $E$. coli infection on the clinical conditions (growth performance, body temperature, fecal score) and axial movement of infected piglets. Our results revealed that oral inoculation of $S$. enteritidis and $E$. coli significantly reduced the growth rate and feed intake of weaned piglets, whereas it increased the feed conversion ratio. Consistent with our results, several studies have reported depressed growth in weaned piglets in response to oral infection with $E$. coli or Salmonella (Krsnik et al., 1999; Balaji et al., 2000; Burkey et al., 2004; Almeida et al., 2013). The reduction in feed intake and growth rate is also consistent with sicknessinduced lack of appetite, which is an expected sickness
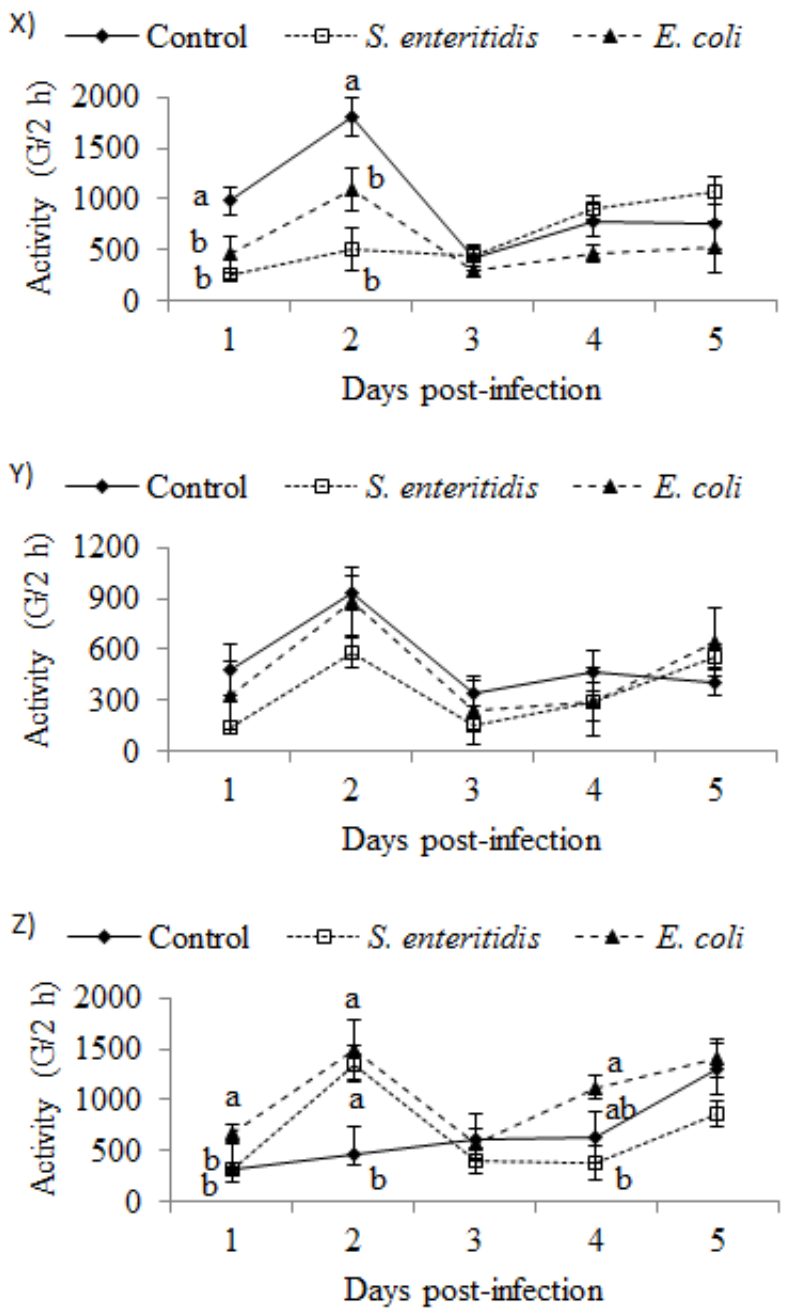

Figure 8. Effects of Salmonella enteritidis and Escherichia coli infection on the night (22:00 to 24:00) axial movements (X, Y, and $\mathrm{Z}$ axis) of weaned piglets. Lines within a time period not sharing a common letter are significantly different $(\mathrm{p}<0.05)$.

behavior associated with the innate immune system in host defense (Johnson, 2002). Bacterial infections increase synthesis of cytokines (Volf et al., 2012), which decreases feed intake (Johnson, 2002). The lower ear temperature of the SE and EC group piglets may lie in their lower metabolic rate due to lower feed intake and growth rate compared to the control group. Consistent to our result, Van Dijk et al. (2002) also reported lower rectal temperature in weaned piglets infected with $E$. coli. Although there was no significant difference, the fecal consistency score of infected piglets was numerically higher (soft feces to mild diarrhea) than the control piglets which may be responsible for lower feed intake (Van Beers-Schreurs et al., 1992).

Behavioral signs have long been considered indicative of illness. The most commonly recognized behavioral patterns of animals at the onset of infectious diseases are lethargy, depression, anorexia, and reduced grooming (Hart, 1988). In this experiment, we measured the axial movement 
of weaned piglets experimentally infected with $S$. enteritidis or $E$. coli using a 3 -axis acceleration sensor. The feasibility of using the acceleration sensor to monitor animal movement has been investigated in many previous studies (Okada et al., 2010; Reith and Hoy, 2011). However, to the best of our knowledge, this is the first study to investigate the axial movement of experimentally infected weaned piglets. Based on the X-axis movement, both infected groups of piglets showed lower movement than control piglets. This can be explained by the reduced feed intake and lower level of motivation energy as a result of bacterial infection (Ivoš and Krsnik, 1979). The Y-axis movement did not differ among treatment groups, except during early morning, when the movement was highest in the E. coli infected group. During the first 3 days post infection (day 1 significantly higher), the Z-axis movements of infected groups were higher than those of the control group, while they were lower than or the same as the control group during the last 2 days. The infected pigs may have discomfort in their stomach due to bacterial infection and diarrhea, which increase the Z-axis (up and down) movement during the first 3 days of experiment. However, development of registrant may help the piglets to overcome the stress and reduce their $\mathrm{Z}$-axis movement during the last 2 days. These results indicate that oral infection with Salmonella and E. coli organisms significantly altered the axial movement of inoculated piglets relative to those recorded in non-inoculated piglets. These findings are in accordance with those reported by Krsnik et al. (1999) and Rostagno et al. (2011), who found altered behavior in response to E. coli and Salmonella infection in piglets.

\section{CONCLUSION}

Failure of the early detection of disease results in increased financial losses for farms and the spread of disease, which can lead to national crises. In this manuscript, we investigated a livestock activity monitoring system using an acceleration sensor for early detection of any expected disease. We found that experimental infection with Salmonella and E. coli resulted in poor growth rate and feed intake. The acceleration results showed distorted movement in infected piglets when compared with non-infected piglets. Overall, the altered movement of infected piglets compared to control piglets was the result of pathogenic infection, and the wireless acceleration sensor could be successfully employed for early detection of illness.

\section{CONFLICT OF INTEREST}

We certify that there is no conflict of interest with any financial organization regarding the material discussed in the manuscript.

\section{ACKNOWLEDGMENTS}

This research was supported by the MSIP (Ministry of Science, ICT and Future Planning), Korea, under the CITRC (Convergence Information Technology Research Center) support program (IITP-2015-H8601-15-1007) supervised by the IITP (National IT Industry Promotion Agency) and iPET (PJ312036-3, Korea Institute of Planning and Evaluation for Technology in Food, Ministry for Food, Agriculture, Forestry and Fisheries, Republic of Korea).

\section{REFERENCES}

Almeida, J. A. S., Y. Liu, M. Song, J. J. Lee, H. R. Gaskins, C. W. Maddox, O. Osuna, and J. E. Pettigrew. 2013. Escherichia coli challenge and one type of smectite alter intestinal barrier of pigs. J. Anim. Sci. Biotechnol. 4:52.

Balaji, R., K. J. Wright, C. M. Hill, S. S. Dritz, E. L. Knoppel, and J. E. Minton. 2000. Acute phase responses of pigs challenged orally with Salmonella typhimurium. J. Anim. Sci. 78:18851891.

Burkey, T. E., S. S. Dritz, J. C. Nietfeld, B. J. Johnson, and J. E. Minton. 2004. Effect of dietary mannanoligosaccharide and sodium chlorate on the growth performance, acute-phase response, and bacterial shedding of weaned pigs challenged with Salmonella enterica serotype Typhimurium. J. Anim. Sci. 82:397-404.

DeVaul, R. W. and S. Dunn. 2001. Real-time motion classification for wearable computing applications. Technical report, MIT Media Laboratory, Cambridge, MA, USA http://www.media.mit.edu/wearables/mithril/realtime.pdf Accessed December 7, 2001.

Foerster, F., M. Smeja, and J. Fahrenberg. 1999. Detection of posture and motion by accelerometry: A validation in ambulatory monitoring. Comput. Hum. Behav. 15:571-583.

Greiner, L. L., T. S. Stahly, and T. J. Stabel. 2000. Quantitative relationship of systemic virus concentration on growth and immune response in pigs. J. Anim. Sci. 78:2690-2695.

Hart, B. L. 1988. Biological basis of the behavior of sick animals. Neurosci. Biobehav. Rev. 12:123-137.

Ivoš, J., B. Krsnik, and S. Kovačević. 1981. Ecology and production in pig-breeding. Stočarstvo 35:379-416.

Ivoš, J. and B. Krsnik. 1979. Some observations of the impact of noise on poultry and swine. Veterinaria (Sarajevo) 2:165-175.

Johnson, R. W. 2002. The concept of sickness behavior: a brief chronological account of four key discoveries. Vet. Immunol. Immunopathol. 87:443-450.

Krsnik, B., R. Yammine, Ž. Pavičić, T. Balenović, B. Njari, I. Vrbanac, and I. Valpotić. 1999. Experimental model of enterotoxigenic Escherichia coli infection in pigs: potential for an early recognition of colibacillosis by monitoring of behavior. Comp. Immun. Microbiol. Infect. Dis. 22:261-273.

Marquardt, R. R., L. Z. Jin, J. W. Kim, L. Fang, A. A. Frohlich, and S. K. Baidoo. 1999. Passive protective effect of egg-yolk antibodies against enterotoxigenic Escherichia coli K88+ infection in neonatal and early-weaned piglets. FEMS Immunol. Med. Microbiol. 23:283-288.

Mepham, T. B. 2000. The role of food ethics in food policy. Proc. 
Nutr. Soc. 59:609-618.

Nadimi, E. S., R. N. Jørgensen, V. Blanes-Vidal, and S. Christensen. 2012. Monitoring and classifying animal behavior using ZigBee-based mobile ad hoc wireless sensor networks and artificial neural networks. Comput. Electron. Agric. 82:4454.

Nardone, A., G. Zervas, and B. Ronchi. 2004. Sustainability of small ruminant organic systems of production. Lives. Product. Sci. 90:27-39.

Okada, H., K. Suzuki, T. Kenji, and T. Itoh. 2010. Avian influenza surveillance system in poultry farms using wireless sensor network. In: Proceedings of the 2010 Symposium on Design Test Integration and Packaging of MEMS/MOEMS (DTIP), Seville, Spain. pp. 253-258.

Pijpers, A., E. J. Schoevers, H. van Gogh, L. A. van Leengoed, I. J. Visser, A. S. van Miert, and J. H. Verheijden. 1991. The influence of disease on feed and water consumption and on pharmacokinetics of orally administered oxytetracycline in pigs. J. Anim. Sci. 69:2947-2954.

Reith, S. and S. Hoy. 2011. Analysis of physical activity, rumination and body weight of dairy cattle during oestrus using sensor-aided systems. In: Proceedings of 8th EFITA/WCCA Conference, Prague, Czech Republic. pp. 107115.
Rostagno, M. H., S. D. Eicher, and D. C. Lay Jr. 2011. Immunological, physiological, and behavioral effects of Salmonella enterica carriage and shedding in experimentally infected finishing pigs. Foodborne Pathog. Dis. 8:623-630.

Schwartz, K. J. 1991. Salmonellosis in swine. Compend. Contin. Educ. Pract. Vet. 13:139-147.

Van Beers-Schreurs, H. M. G., L. Vellenga, Th. Wensing, and H. J. Breukink. 1992. The pathogenesis of the post-weaning syndrome in weaned piglets: A review. Vet. Q. 14:29-34.

Van Dijk, A. J., P. M. M. Enthoven, S. G. C. Van den Hoven, M. M. M. H. Van Laarhoven, T. A. Niewold, M. J. A. Nabuurs, and A. C. Beynen. 2002. The effect of dietary spray-dried porcine plasma on clinical response in weaned piglets challenged with a pathogenic Escherichia coli. Vet. Microbiol. 84:207-218.

Volf, J., H. Stepanova, J. Matiasovic, K. Kyrova, F. Sisak, H. Havlickova, L. Leva, M. Faldyna, and I. Rychlik. 2012. Salmonella enterica serovar Typhimurium and Enteritidis infection of pigs and cytokine signalling in palatine tonsils. Vet. Microbiol. 156:127-135.

Weary, D. M., J. M. Huzzey, and M. A. G. von Keyserlingk. 2009. Board-invited review: using behavior to predict and identify ill health in animals. J. Anim. Sci. 87:770-777. 